\title{
Long term consequences of regressed coronary aneurysms after Kawasaki disease: vascular wall morphology and function
}

\author{
M Iemura, M Ishii, T Sugimura, T Akagi, H Kato
}

\begin{abstract}
Objectives-To investigate the long term consequences of regressed aneurysms after Kawasaki disease, using follow up coronary angiography; to assess the vascular wall morphology at the site of the aneurysms by intravascular ultrasound imaging; and to evaluate the function of the affected vessels using intracoronary infusions of acetylcholine and isosorbide dinitrate.

Design-33 patients were studied, 27 with previous Kawasaki disease and six with congenital heart disease. All Kawasaki disease patients were followed for more than 10 years from disease onset. The 33 patients comprised four groups: group 1 included 13 Kawasaki disease patients with a total of 23 sites of regressed large sized ( $\geqslant 4 \mathrm{~mm}$ ) coronary aneurysms; group 2 included 13 Kawasaki disease patients with 22 sites of regressed small sized $(<4 \mathrm{~mm})$ coronary aneurysms (four patients had sites of both large and small sized aneurysms); group 3 included a further five Kawasaki disease patients with 25 normal coronary angiography sites in the acute stage of Kawasaki disease; and group 4 comprised the six patients with congenital heart disease as controls, with a total of 27 normal coronary angiography sites. During coronary angiography, $15 \mu \mathrm{g}$ of acetylcholine and $0.5 \mathrm{mg}$ isosorbide dinitrate were infused into the coronary artery. The luminal diameter at the sites was measured using a cine-videodensitometric analyser, to assess the distensibility of the coronary artery wall.

Results-Coronary angiography in all 22 patients in groups 1 and 2 and in all the patients in group 3 was normal, with no stenoses and no irregularity of the arterial wall. However, the intravascular ultrasound imaging in groups 1 and 2 showed various degrees of the intimal thickening. In groups 1 and 2, there was significantly more vascular constriction with acetylcholine, and poorer dilatation with isosorbide dinitrate than in groups 3 or 4 (each $\mathrm{p}<0.05$, respectively). There was no difference between group 3 and group 4 in response to either acetylcholine or isosorbide dinitrate,

Conclusions-There is evidence of persisting abnormal vascular wall morphology and vascular dysfunction at the site of regressed coronary aneurysms in patients with previous Kawasaki disease. These patients should be counselled to avoid potential risk factors for atherosclerosis, and long term follow up is needed into adult life.

(Heart 2000;83:307-311)
\end{abstract}

Keywords: Kawasaki disease; coronary aneurysm; atherosclerosis; intravascular ultrasound

About 30 years have passed since Kawasaki disease was first described in Japan. ${ }^{12}$ Approximately $50 \%$ of coronary aneurysms occurring in the acute stage of this disease were shown to regress within several years, and normal angiographic findings were demonstrated..$^{3-5}$ Pathologically, there was marked thickening in the intima, caused mainly by proliferation of smooth muscle cells. ${ }^{6}$ This proliferation resulted in a normal sized lumen in the aneurysmal coronary artery. However, the long term prognosis and natural history of Kawasaki disease, particularly in patients with regressed coronary aneurysms or with normal coronary arteries, remain uncertain. On the basis of our own $^{3-11}$ and other ${ }^{12-14}$ previous studies, we felt there was a need to examine prospectively the long term consequences of regressed coronary aneurysms and normal coronary arteries after Kawasaki disease. Intravascular ultrasound imaging of coronary arteries now allows detailed visualisation of coronary wall morphology and the healing process in patients with Kawasaki disease. ${ }^{1014}$ Acetylcholine chloride, an endothelium dependent vasodilator, is believed to dilate normal coronary arter- ies by promoting the release of a vasorelaxant substance from the endothelium, probably nitric oxide (NO).$^{15-20}$ In coronary atherosclerosis, muscarinic cholinergic vasodilatation is impaired, and paradoxical vasoconstriction is induced by acetylcholine. ${ }^{15-19}$ Conversely, isosorbide dinitrate is an endothelium independent vasodilator that causes NO release as it is metabolised. ${ }^{21}{ }^{22}$ Our aims in this study were first, to investigate the long term consequences of regressed and normal coronary arteries after Kawasaki disease, using follow up coronary angiography; second, to assess vascular wall morphology at the site of the previous aneurysms by intravascular ultrasound imaging; and third, to evaluate vascular function at sites of previous aneurysms and at sites where the vessels had been normal during the acute phase of the disease, using intracoronary infusions of acetylcholine and isosorbide dinitrate.

\section{Methods}

PATIENTS

From September 1996 to January 1998, we studied 33 patients in all. These included 27 patients with Kawasaki disease who had been 
followed up for more than 10 years since the acute stage of the disease, and six patients with mild congenital heart disease, who comprised a control group. The patients had suffered from Kawasaki disease at ages ranging from three months to 5.8 years (mean (SD), 1.6 (1.4) years) and had been followed for 10 to 16 years (mean 14.4 (1.6) years) at Kurume University Hospital.

Coronary angiography was first performed in each Kawasaki disease patient just after the acute stage of illness to evaluate coronary artery lesions. These 27 patients comprised 22 in whom coronary aneurysms had regressed to normal coronary angiographic findings within two years (male to female ratio, 11/11; age, 15.9 (2.0) years; follow up period, 14.4 (1.1) years), and five with normal coronary angiographic findings in the acute stage of Kawasaki disease (male to female ratio, 5/0; age, 16.3 (2.1) years; follow up period, 14.7 (3.1) years). All patients with Kawasaki disease had received treatment with aspirin $(30 \mathrm{mg} / \mathrm{kg}$ daily in the acute phase; $5 \mathrm{mg} / \mathrm{kg}$ daily in the convalescent phase). No high dose gamma globulin was given to the patients in this study, as all the patients suffered from the disease before the first report of this treatment. ${ }^{23}$ As controls, six age matched patients with mild congenital heart disease were included in the study. One had a small ventricular septal defect and five an atrial septal defect (male to female ratio, 5/1; age, 13.7 (1.8) years). All control patients had normal coronary arteries on coronary angiography. No patient had documented hypertension, ${ }^{24}$ hypercholesterolaemia, ${ }^{25}$ or a family history of diabetes mellitus, and there were no smokers.

\section{CORONARY ANGIOGRAPHY AND VASCULAR}

FUNCTION STUDIES

Cineangiography of the left and right coronary arteries was performed by injecting iohexol manually with ECG monitoring. After the initial coronary angiogram, we infused $15 \mu \mathrm{g}$ of acetylcholine chloride (Daiichi Seiyaku, Tokyo, Japan), diluted in $5 \mathrm{ml}$ of warm $0.9 \%$ saline solution, into the right or left coronary artery over 30 seconds. ${ }^{11}$ This dose of acetylcholine corresponded to an estimated blood concentration of $3.0 \times 10^{-6} \mathrm{M} \cdot{ }^{11}$ A second coronary angiography procedure was performed in both coronary arteries 30 seconds after the completion of the acetylcholine infusion. After the second coronary angiogram, we next infused $0.5 \mathrm{mg}$ of isosorbide dinitrate (Eisai, Tokyo, Japan), diluted in $5 \mathrm{ml}$ of warm $0.9 \%$ saline solution, into the same coronary artery over 30 seconds. ${ }^{911} \mathrm{~A}$ third coronary angiography pro-

Table 1 Details of the study groups

\begin{tabular}{lllll}
\hline Patients & Initial aneurysm size & $\begin{array}{l}\text { Number of } \\
\text { sites }\end{array}$ & Age (years) & $\begin{array}{l}\text { Follow up period } \\
\text { (years) }\end{array}$ \\
\hline Kawasaki disease & & & \\
Group 1 & $\geqslant 4 \mathrm{~mm}$ & 23 & $16.3(2.1)$ & $14.5(1.1)$ \\
Group 2 & $<4 \mathrm{~mm}$ & 22 & $15.9(2.0)$ & $14.4(1.1)$ \\
Group 3 & No coronary artery lesion & 25 & $16.3(2.1)$ & $14.7(3.1)$ \\
Control & Normal coronary artery & 27 & $13.7(1.8)$ & \\
Group 4 & 27 & & \\
\hline
\end{tabular}

${ }^{\star}$ Mean (SD). cedure was performed one minute after the completion of the isosorbide dinitrate infusion.

SITES OF CORONARY ARTERY LESIONS STUDIED In this study, we analysed numbers of coronary artery sites rather than numbers of patients, because various sizes of coronary aneurysms were presented at the same time in some patients. We divided the patients into four groups depending on the size of the initial coronary aneurysm on first coronary angiogram (table 1). ${ }^{26}$ Group 1 (13 patients with Kawasaki disease; 23 sites; mean (SD) age, 16.3 (2.1) years) had regressed coronary aneurysms that were initially $4 \mathrm{~mm}$ or more in diameter. Group 2 (13 patients with Kawasaki disease; 22 sites; age 15.9 (2.0) years) also had regressed coronary aneurysms, but these were initially less than $4 \mathrm{~mm}$ in diameter. Four patients had sites of both large and small aneurysms. Group 3 (five patients with Kawasaki disease; 25 sites; age, 16.3 (2.1) years) had normal coronary angiography findings during the acute stage of the disease. Group 4 (six patients with congenital heart disease; 27 sites; age 13.7 (1.8) years) served as the age matched control group. There were no significant differences among the four groups for age, sex, heart rate, systolic blood pressure, serum cholesterol, and coronary risk factors.

The study protocol was approved by the Kurume University School of Medicine ethics committee, and written informed consent was obtained from the patients and their parents.

\section{QUANTITATIVE CORONARY ANGIOGRAPHIC}

ANALYSIS

Paired, identical, orthogonal views before and after both acetylcholine and isosorbide dinitrate infusion were chosen for quantitative coronary angiographic analysis, using an image analysing system (Cardio 500, Kontron Elektronik Corporation, Germany). End diastolic frames were selected for analysis. Each lesion was measured three times and the mean values were used for statistical analysis. In groups 1 and 2, we measured the luminal diameter at the site of the initial aneurysms, and in groups 3 and 4 we measured the diameter at segments 1 , $2,3,5$, and 6 , where coronary aneurysms in Kawasaki disease are likely to develop. ${ }^{3}$ The change in the coronary artery diameter was calculated by the following formula: per cent change $=($ diameter after infusion - diameter before infusion)/diameter before infusion $\times$ 100 . To evaluate the effect of observer variability on the quantitative coronary angiography, two independent observers (MI and TS) analysed 15 randomly selected patients at different times with the same image analysing system; each observer individually selected the frames to measure and had no knowledge of the results obtained by the other observer.

INTRAVASCULAR ULTRASOUND IMAGING PROTOCOL AND ANALYSIS

Intravascular ultrasound examination was performed using $30 \mathrm{MHz}$ catheters (3.5 F, Boston Scientific Corporation, Boston, Massachusetts, USA). The transducer was advanced distal to 


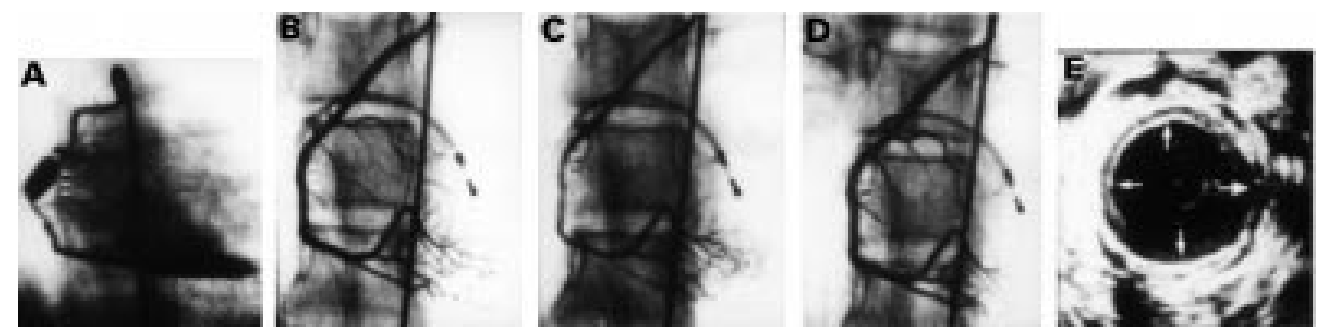

Figure 1 Coronary angiograms and intravascular ultrasound images of a patient in group 1. First angiography 55 days after onset of Kawasaki disease (A). Follow up angiography 14 years after the onset of Kawasaki disease: in steady state (B); after intracoronary infusion of acetylcholine (C); and after intracoronary infusion of isosorbide dinitrate (D); intravascular ultrasonographic images at the regressed site of coronary artery $(E)$.

the target segment and then withdrawn manually. ${ }^{10}$ The intravascular ultrasound images were recorded onto videotape (S-VHS). Fifteen patients in groups 1 and 2, all five patients in group 3, and all six patients in group 4 were examined using intravascular ultrasound imaging (group 1: 12 sites in nine patients; group 2: 11 sites in six patients; group 3: 13 sites in five patients; group 4: 14 sites in six patients). We carefully examined the morphology of the coronary artery wall on intravascular ultrasound images at the site of the initial aneurysm in groups 1 and 2, and in segments $1,2,3,5$, and 6 in groups 3 and 4 . We then calculated the thickness of the intimamedia complex. ${ }^{1014}$

\section{STATISTICAL ANALYSIS}

Analysis of variance for repeated measurement with Scheffe's F test was used to compare groups. The Student $t$ test was used for two way comparison of data. A probability (p) value of $<0.05$ was considered significant.

\section{Results}

FOLLOW UP ANGIOGRAPHIC FINDINGS

None of the regressed coronary aneurysms in the 22 patients in groups 1 and 2 developed stenotic lesions in the long term follow up period (fig 1, A and B). Coronary angiography was completely normal, with no evidence of irregularity of the vessel wall or calcification. There was also no evidence of collateral circulation. Left ventricular volume and ejection fraction were within normal limits. All patients in groups 3 and 4 also had normal coronary angiography findings and normal left ventricular function.

VASCULAR WALL MORPHOLOGY ON INTRAVASCULAR ULTRASOUND IMAGING

Fifty sites in 26 patients were examined using intravascular ultrasound imaging (group 1: 12 sites in nine patients; group 2: 11 sites in six patients; group 3: 13 sites in five patients; group 4: 14 sites in six patients). The intravascular ultrasound images in groups 1 and 2 showed various degrees of intimal thickening but no evidence of calcification. In group 1, at sites of regressed moderate to large sized coronary aneurysms there was a circumferential three layered appearance, with an increased bright inner layer from the intimal hyperplasia and a normal media, but with no evidence of calcification (fig 1E). There was a significant difference in the thickness of the intima-media
Table 2 Thickness of intima-media complex, group $1 v$ group 2

\begin{tabular}{lll}
\hline & $\begin{array}{l}\text { Initial aneurysm } \\
\text { size }\end{array}$ & $\begin{array}{l}\text { Intima-media } \\
\text { complex }(\mathrm{mm})\end{array}$ \\
\hline Group 1 & $\geqslant 4 \mathrm{~mm}$ & $0.59(0.13)^{\star}$ \\
Group 2 & $<4 \mathrm{~mm}$ & $0.29(0.06)$ \\
\hline
\end{tabular}

Values are mean (SD).

${ }^{\star} \mathrm{p}<0.05$ between group 1 and group 2

complex between groups 1 and 2 (table 2). However, in all intravascular ultrasound images in group 3, the coronary artery wall echo had a single layered appearance, similar to that in the control patients in group 4.

\section{VASCULAR FUNCTION}

The mean (SD) change in the coronary artery diameter after infusion of acetylcholine was $-15.9(14.5) \%$ in group 1 (fig 1C); -1.8 $(8.3) \%$ in group $2 ; 11.3(4.6) \%$ in group 3 ; and $15.5(5.6) \%$ in group 4 (fig 2). The change in the coronary artery diameter after infusion of isosorbide dinitrate was $7.7(5.6) \%$ in group 1 (fig 1D); 11.0 (5.5)\% in group $2 ; 18.0(8.2) \%$ in group 3; and 16.6 (7.1)\% in group 4 . In groups 1 and 2 , there was more vascular constriction with acetylcholine and less dilatation with isosorbide dinitrate than in groups 3 or 4 (both $p<0.05$, fig 2 ). A difference in the response to acetylcholine was also found between groups 1 and 2 ( $p<0.05$, fig 2$)$. Group 3 showed no significant difference from

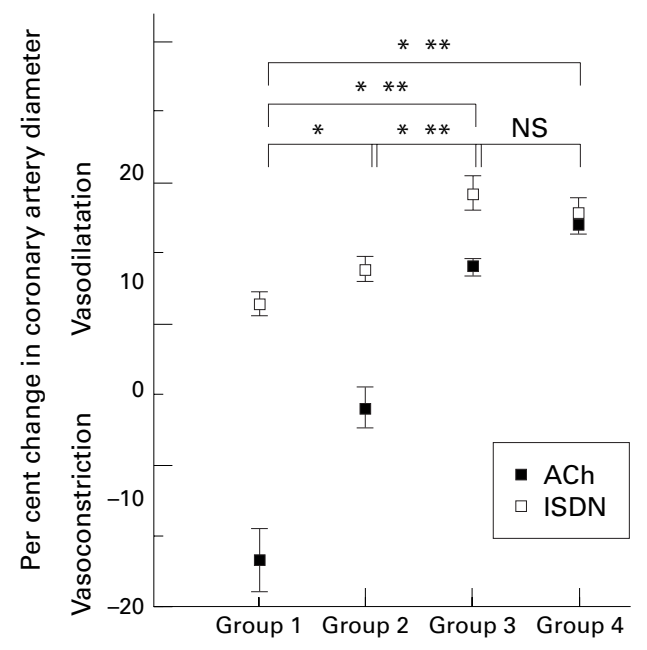

Figure 2 Mean percentage change in the diameter of the coronary artery after intracoronary infusion of acetylcholine $(A C h)$ and isosorbide dinitrate (ISDN) in each group. Error bars $=S E M .{ }^{\star} p<0.05$ (ACh); ${ }_{\star *} p<0.05$ (ISDN). 
the control group 4 in response to the infusion of either acetylcholine or isosorbide dinitrate (fig 2).

INTEROBSERVER VARIABILITY

An excellent correlation was found between observers 1 and 2 in determining the percentage change in coronary artery diameter $(r=0.97, \mathrm{p}<0.001)$. The mean interobserver difference for the percentage change was 0.07 $(0.01) \%$.

\section{Discussion}

We have previously reported that more than $50 \%$ of coronary aneurysms occurring in Kawasaki disease regress within a period of years, and have shown normal angiographic findings on follow up. ${ }^{3-5}$ However, important questions remain concerning the long term outcome of regressed coronary aneurysms, and indeed of apparently normal coronary arteries, after Kawasaki disease.

LONG TERM FOLLOW UP CORONARY

ANGIOGRAPHIC FINDINGS AFTER KAWASAKI

DISEASE

Whether regression might reverse and eventually develop into stenosis is uncertain at present. Suzuki et al considered that the development of stenosis was possible in the long term. ${ }^{13}$ However, in our present study, none of the regressed coronary aneurysms in 22 patients developed stenotic lesions over a long follow up period, and the coronary angiographic findings were completely normal. Our previous histological study showed well regenerated endothelium and marked intimal thickening, without evidence of recanalisation of organised thrombi in resected coronary aneurysms. ${ }^{6}$ These events may represent the healing process of angiitis in Kawasaki disease. The histological findings supported our clinical results. Our current findings indicate that stenotic lesions do not develop within one or two decades in patients who have shown regression of coronary aneurysms. All patients with Kawasaki disease in our series had received aspirin, $30 \mathrm{mg} / \mathrm{kg}$ daily, during the acute stage of illness, reduced to $5 \mathrm{mg} / \mathrm{kg}$ daily while abnormal coronary angiographic findings were still present. ${ }^{27}{ }^{28}$ This anticoagulant treatment may have contributed to reducing the late effects of Kawasaki disease vasculitis. Although the regulatory factors that limit the progression of the disease are unknown at present, the absence of progression to stenosis may reflect coronary haemodynamic factors such as shear stress.

No high dose gamma globulin was given to the patients in this study, but this did not appear to have had any long term adverse effect. The long term effects of gamma globulin treatment on the coronary arteries - that is, the incidence of regression, the occurrence of stenosis or obstruction, and the development of aneurysm-are not clear at present. Further studies are necessary to clarify the long term effects of high dose gamma globulin treatment.
LONG TERM VASCULAR WALL MORPHOLOGY AFTER KAWASAKI DISEASE

In this study we found various degrees of intimal thickening but normal media on intravascular ultrasound imaging of the sites of regressed aneurysms. These findings suggest that there has been proliferation of smooth muscle cells as part of the healing process after the vasculitis that occurs in the acute stage of Kawasaki disease. ${ }^{6}$ Our intravascular ultrasound findings were similar to those in arteriosclerosis. In contrast, intravascular ultrasound imaging in patients whose coronary arteries had been apparently normal in the acute phase of the disease showed a single layered appearance. The findings in these patients were similar to those in normal children and adolescents. $^{10}$

LONG TERM VASCULAR FUNCTION AFTER

KAWASAKI DISEASE

We evaluated vascular function after Kawasaki disease by intracoronary injection of acetylcholine and isosorbide dinitrate. Acetylcholine is believed to dilate normal blood vessels by promoting the release of an endothelium derived relaxing factor such as NO. ${ }^{16-20}$ In contrast, after the endothelium is removed experimentally, acetylcholine constricts the blood vessels. In coronary atherosclerosis, muscarinic cholinergic vasodilatation is impaired and paradoxical vasoconstriction is induced by acetylcholine. ${ }^{16-20}$ Conversely, isosorbide dinitrate is an endothelium independent vasodilator which causes NO release as it is metabolised. ${ }^{2122}$ At the sites of regressed aneurysms acetylcholine caused significant vasoconstriction (group 1) and mild vasoconstriction or weak dilatation (group 2). The regressed coronary aneurysms were dilated by isosorbide dinitrate but to only a mild degree. These results suggest that the regressed coronary aneurysms may have endothelial dysfunction, with impairment of endothelium dependent vasodilatation. There may also be smooth muscle dysfunction, as the response to isosorbide dinitrate at sites of regressed coronary aneurysms was only small. Together with the intravascular ultrasound findings, the abnormal functional behaviour of the coronary artery may have resulted from pathological changes occurring after Kawasaki disease. Our previous study showed that the response to isosorbide dinitrate at sites of persistent coronary aneurysms was significantly less than at the same sites in normal coronary arteries. ${ }^{10}$ Another previous study in our institution found that persistent coronary aneurysms showed almost no change in calibre during acetylcholine infusion. ${ }^{11}$ These findings suggest that the region of coronary artery containing persistent aneurysms may be stiffer than normal owing to endothelial or smooth muscle dysfunction.

We found that coronary arteries that had appeared normal during the acute phase of Kawasaki disease showed no significant difference from control in response to either acetylcholine or isosorbide dinitrate infusion. This suggests that such arteries have normal 
endothelial and smooth muscle function in the long term. Mitani et al showed that after Kawasaki disease a normal region of the coronary artery constricted during acetylcholine infusion. ${ }^{12}$ However, there were several differences between our present study and theirs. For example, their patients did not have completely normal coronary arteries in the acute stage, only a normal left anterior descending coronary artery. ${ }^{12}$ In our study, on the other hand, group 3 patients had completely normal coronary arteries (both right and left) in the acute stage. Moreover, the time interval between Kawasaki disease onset and the follow up investigation in Mitani's study was shorter than in our study. ${ }^{12}$ Pathological studies have shown that an inflammatory cell infiltrate may still be present in the coronary arteries as long as six months after the acute phase of Kawasaki disease. ${ }^{29}{ }^{30}$ In our present study, we investigated the patients only after an interval of more than 10 years after disease onset to eliminate any direct effects of acute Kawasaki disease vasculitis.

\section{CLINICAL IMPLICATIONS}

Approximately $50 \%$ of patients who had coronary aneurysms show regression on follow up coronary angiography. ${ }^{4}$ This is a characteristic phenomenon in Kawasaki vasculitis. ${ }^{5}$ Coronary angiography in all 22 patients with regressed coronary aneurysms after Kawasaki disease showed completely normal findings over the long term follow up period. However, our study showed that these vessels had marked thickening in the intima on intravascular ultrasound examination, together with endothelial dysfunction similar to that occurring in early atherosclerotic lesions. Therefore regressed aneurysms in Kawasaki vasculitis may be a long term coronary risk factor, and atherosclerotic lesions might develop in adult life. ${ }^{31}$ These patients should be followed up carefully and advised to avoid other atherogenic risk factors such as smoking, obesity, hypertension, and hypercholesterolaemia. In addition, these patients might benefit from term antiplatelet drug treatment such as low dose aspirin to improve endothelial function and prevent the development of atheromatous coronary artery disease at a late stage. In the future, prospective research is required to assess the potential benefit of therapeutic approaches in Kawasaki disease patients, including risk factor control and antiplatelet treatment.

Our study showed that patients with Kawasaki disease who had normal coronary arteries in the acute phase had normal vascular wall morphology and vascular function in the long term. We think such patients are unlikely to have any late morbidity.

\section{CONCLUSIONS}

Long term follow up seems necessary both for patients with persistent coronary lesions and for those with regressed coronary aneurysms. Such follow up should focus on the possible development of premature atherosclerosis and should include advice to the patients to avoid atherogenic risk factors.
Supported in part by grants in aid 10470183 and 09770586 from the Ministry of Education, Science of Culture and Vehara Memorial Foundation, Japan.

1 Kawasaki T. Acute febrile mucocutaneous syndrome with the lymphoid involvement with specific desquamation of the

2 Kawasaki T, Kosaki F, Okawa S, et al. A new infantile acute febrile mucocutaneous lymph node syndrome (MLNS) prevailing in Japan. Pediatrics 1974;54:271-6.

3 Kato H, Ichinose E, Yoshioka F, et al. Fate of coronary aneurysms in Kawasaki disease: serial coronary angiography and long-term follow-up study. Am f Cardiol 1981; 49:1758-66.

4 Kato $\mathrm{H}$, Sugimura $\mathrm{T}$, Akagi $\mathrm{T}$, et al. Long-term consequences of Kawasaki disease: a 10 to 21 year follow-up study of 594 patients. Circulation 1996;94:1379-85.

5 Kato H, Koike S, Yamamoto $M$, et al. Coronary aneurysms in infants and young children with acute febrile mucocutain infants and young children with acute febrile mucocuta

6 Sasaguri Y, Kato H. Regression of aneurysms in Kawasaki disease: a pathological study. F Pediatr 1982;100:225-31.

7 Kato H, Inoue O, Akagi T. Kawasaki disease: cardiac problems and management. Pediatr Rev 1988;9:209-17.

8 Kato H, Akagi T, Sugimura T, et al. Kawasaki disease. Coron Artery Dis 1995;6:194-206.

9 Sugimura $\mathrm{T}$, Kato $\mathrm{H}$, Inoue $\mathrm{O}$, et al. Vasodilatory response of the coronary arteries after Kawasaki disease: evaluation by intracoronary injection of isosorbide dinitrate. $\mathcal{F}$ Pediatr 1992;121:684-8.

10 Sugimura $\mathrm{T}$, Kato $\mathrm{H}$, Inoue $\mathrm{O}$, et al. Intravascular ultrasound of coronary arteries in children: assessment of the wall morphology and the lumen after Kawasaki disease. Circulation 1994;89:258-65.

11 Yamakawa R, Ishii M, Sugimura T, et al. Coronary endothelium dysfunction after Kawasaki disease: evaluation by intracoronary injection of acetylcholine. $7 \mathrm{Am}$ Coll Cardiol 1998;31:1074-80

12 Mitani Y, Okuda Y, Shimpo H, et al. Impaired endothelial function in epicardial coronary arteries after Kawasaki disease. Circulation 1997;96:454-61.

13 Suzuki A, Kamiya T, Arakaki Y, et al. Fate of coronary arterial aneurysms in Kawasaki disease. Am f Cardiol 1994;74: $822-4$.

14 Suzuki A, Yamaguchi M, Kimura K, et al. Functional behavior and morphology of the coronary artery wall in
patients with Kawasaki disease assessed by intravascular patients with Kawasaki disease assessed by
ultrasound. 7 Am Coll Cardiol 1996;27:291-6.

15 Ishii $\mathrm{M}$, Kato $\mathrm{H}$, Kawano $\mathrm{T}$, et al. Evaluation of pulmonary arterial histropathologic findings in congenital heart disease: an in vitro study using intravascular ultrasound imaging. I Am Coll Cardiol 1995;26:272-6.

16 Ludmer PL, Selwyn AP, Shook TL, et al. Paradoxical vasoconstriction induced by acetylcholine in atherosclerotic coronary arteries. N Engl f Med 1986;315:1046-51.

17 Palmer RMJ, Ferrige AG, Moncada S. Nitric oxide release accounts for the biological activity of endothelium-derived relaxing factor. Nature 1987;327:524-6.

18 Hodgson JM, Marshall JJ. Direct vasoconstriction and endothelium-dependent vasodilatation: mechanisms of acetylcholine diameter in patients with nonstenotic coronary arteries. Circulation 1989;79:1043-51.

19 Egashira K, Inou T, Hirooka Y, et al. Evidence of impaired endothelium-dependent coronary vasodilatation in patients with angina pectoris and normal coronary angiogram. N Engl F Med 1993;328:1659-64.

20 Anderson TJ, Meredith IT, Ganz P, et al. Nitric oxide and nitrovasodilators: similarities, differences and potential interactions. $7 \mathrm{Am}$ Coll Cardiol 1994;24:555-66.

21 Feelisch $M$. Biotransformation to nitric oxide of organic nitrates in comparison to other nitrovasodilators. Eur Heart J 1993;14(suppl I):123-32.

22 Harrison DG, Bates JN. The nitrovasodilators: new ideas about old drugs. Circulation 1993;87:1461-7.

23 Furusho K, Kamiya T, Nakano H, et al. High-dose intravenous gamma globulin for Kawasaki disease. Lancet 1984;ii: $1055-8$.

24 Task Force on Blood Pressure Control in Children. Report of the second task force on blood pressure in children. Pediatrics 1987;79:1-25.

25 Corther AJ, Coates PM, Tershakovec AM. Disorders of lipoprotein metabolism and transport. In: Behrman RE, ed. Nelson textbook of pediatrics. Philadelphia, PA: WB ed. Nelson textbook
Saunders, 1992:354.

26 Nakano H, Ueda K, Saito A, et al. Repeated quantitative angiograms in coronary arterial aneurysm in Kawasaki disangiograms in coronary arterial aneury

27 Kato H, Koike S, Yokoyama T. Kawasaki disease: effect of treatment on coronary artery involvement. Pediatrics 1979; 63:175-9

28 Akagi $\mathrm{T}$, Kato $\mathrm{H}$, Inoue $\mathrm{O}$, et al. Salicylate treatment in Kawasaki disease: high dose or low dose? Eur $\mathcal{f}$ Pediatr 1991;150:642-6.

29 Hirose S, Hamashima Y. Morphological observation on the vasculitis in the mucocutaneous lymph node syndrome. Eur f Pediatr 1978;129:17-27.

30 Fujiwara H, Hamashima Y. Pathology of the heart in KawaFujiwara H, Hamashima Y. Pathology of

31 Dhillon R, Clarkson P, Donald AE, et al. Endothelial dysfunction late after Kawasaki disease. Circulation 1996; 94:2103-6. 